\title{
Relativistic Two-Dimensional Harmonic Oscillator Plus Cornell Potentials in External Magnetic and AB Fields
}

\author{
Sameer M. Ikhdair ${ }^{1,2}$ and Ramazan Sever ${ }^{3}$ \\ ${ }^{1}$ Department of Physics, Faculty of Science, An-Najah National University, P.O. Box 7, Nablus, 400 West Bank, Palestine \\ ${ }^{2}$ Department of Electrical and Electronic Engineering, Near East University, 922022 Nicosia, Northern Cyprus, Turkey \\ ${ }^{3}$ Department of Physics, Middle East Technical University, 06531 Ankara, Turkey
}

Correspondence should be addressed to Sameer M. Ikhdair; sameer.ikhdair@najah.edu

Received 13 June 2013; Accepted 7 September 2013

Academic Editor: Shi-Hai Dong

Copyright (c) 2013 S. M. Ikhdair and R. Sever. This is an open access article distributed under the Creative Commons Attribution License, which permits unrestricted use, distribution, and reproduction in any medium, provided the original work is properly cited.

\begin{abstract}
The Klein-Gordon (KG) equation for the two-dimensional scalar-vector harmonic oscillator plus Cornell potentials in the presence of external magnetic and Aharonov-Bohm $(\mathrm{AB})$ flux fields is solved using the wave function ansatz method. The exact energy eigenvalues and the wave functions are obtained in terms of potential parameters, magnetic field strength, $A B$ flux field, and magnetic quantum number. The results obtained by using different Larmor frequencies are compared with the results in the absence of both magnetic field $\left(\omega_{L}=0\right)$ and $\mathrm{AB}$ flux field $(\xi=0)$ cases. Effect of external fields on the nonrelativistic energy eigenvalues and wave function solutions is also precisely presented. Some special cases like harmonic oscillator and Coulombic fields are also studied.
\end{abstract}

\section{Introduction}

The exact solution of Schrödinger equation (SE) and the relativistic wave equations for some physical potentials are very important in many fields of physics and chemistry since they contain all the necessary information for the quantum system under investigation. The hydrogen atom and the harmonic oscillator are usually given in textbooks as two of several exactly solvable problems in both classical and quantum physics [1]. The exact $l$-state solutions of the SE are possible only for a few potentials, and hence approximation methods are used to obtain their solutions. According to the Schrödinger formulation of quantum mechanics, a total wave function provides implicitly all relevant information about the behaviour of a physical system. Hence, if it is exactly solvable for a given potential, the wave function can describe such a system completely. Until now, many efforts have been made to solve the stationary SE with anharmonic potentials in three dimensions (3D) and two dimensions (2D) [2-7] with many applications to molecular and chemical physics. However, the study of SE with some of these potentials in arbitrary dimension $\mathrm{D}$ is also solved in the following (cf.
[8] and the references therein). The study of bound states is fundamental in the understanding of molecular spectrum of a diatomic molecule and provides us with insight into the physical problem under consideration in quantum mechanics [9].

Recently, some authors have studied the bound states of the $l$-wave equations with some typical potentials in the presence of an equal scalar potential $S(r)$ and a vector potential $V(r)$. These potentials include the harmonic oscillator potential $[10,11]$, ring-shaped Kratzer-type potential [12], pseudo-harmonic oscillator potential [13], double ringshaped harmonic oscillator potential [14], and ring-shaped pseudo-harmonic oscillator potential [15-17].

It is well-known that the non-relativistic quantum mechanics is an approximate theory of the relativistic one. However, when a particle moves in a strong potential field, the relativistic effects must be considered which give the corrections for non-relativistic quantum mechanics [18]. Therefore, the motion of spin- 0 and spin- $1 / 2$ particles satisfies the KG and the Dirac equations, respectively. In particular, solutions to relativistic equations play an important role in many aspects of modern physics. For instance, the Dirac 
equation has been used to explain the antinucleon bound in a nucleus [19], deformed nuclei [20], and super deformation [21] and to establish an effective nuclear shell model scheme [22-24], while the KG equation has been used in describing a wide variety of phenomena, which include classical wave systems, such as the displacement of a string attached to an elastic bed [25] and quantum system based on scalar field theories [26].

Recently, the Schrödinger equation is solved exactly for its bound states (energy spectrum and wave functions) [2729 ] to study the spectral properties in a $2 \mathrm{D}$ charged particle (electron or hole) confined by a harmonic oscillator in the presence of external strong uniform magnetic field $\vec{B}$ along the $z$ direction and Aharonov-Bohm (AB) flux field created by a solenoid. The energy levels and the wave functions of an electron confined by 2D harmonic and pseudo-harmonic oscillators have been studied in presence of external fields [30, 31] using the Nikiforov-Uvarov (NU) method. Overmore, it is natural to study the relativistic effects of the external magnetic and $\mathrm{AB}$ flux fields on the KG equation for pseudo-harmonic oscillator potential, especially for a strong coupling by means of the NU method [32]. The 2D solution of Schrödinger equation for the Kratzer potential with and without the presence of a constant magnetic field is studied within the framework of the asymptotic iteration method [33]. The energy eigenvalues are obtained analytically (numerically) for the absence (presence) of uniform magnetic field case. These results have been obtained by using different Larmor frequencies $\left(\omega_{L} \neq 0\right)$ and potential parameters are compared with the results in the absence of magnetic field case $\left(\omega_{L}=\right.$ $0)$. Overmore, the spectral properties of the $2 \mathrm{D}$ Schrödinger equation for the pseudo-harmonic-Coulomb-linear potential are studied using the analytical iteration method [34]. Under spin symmetry, the energy states and wave functions of the Dirac equation for the Killingbeck (harmonic oscillator plus Cornell) potential have been carried out using the wave function ansatz method [35]. The spin and pseudospin symmetry in Dirac equation have been studied in the Killingbeck potential within the context of quasi-exact solution [36]. Further, the energy eigenvalues and normalized eigenfunctions of the radial Schrödinger equation in $N$-dimensional Hilbert space for the quark-antiquark interaction Killingbeck potential have been obtained using the power series technique via a suitable choice of ansatz to the wave function [37, 38].

Very recently, we studied the scalar charged particle exposed to relativistic scalar-vector Killingbeck potentials in presence of magnetic and Aharonov-Bohm flux fields and obtained its energy eigenvalues and wave functions using the analytical exact iteration method [39]. Therefore, the behavior of a spinless relativistic particle moving under the Killingbeck potential in a static magnetic and AB flux fields has not been investigated yet, and in this work, we aim to solve the KG equation in 2D for equal mixture of scalar and vector Killingbeck potentials with and without constant magnetic and $\mathrm{AB}$ flux fields for the first time. We present the exact energy eigenvalues and wave functions of the Killingbeck potential for any $n$ and $m$ quantum numbers in a constant magnetic field with the different Larmor frequencies using the wave function ansatz method [36]. The non-relativistic energy eigenvalues and wave functions of our solution are presented by making an appropriate mapping of parameters. Overmore, special cases of KG for equal scalar-vector Killingbeck potentials are also presented in the presence $\left(\omega_{L} \neq 0\right.$, $\xi \neq 0)$ and absence $\left(\omega_{L}=0, \xi=0\right)$ of uniform fields.

The structure of this paper is as follows. We study effect of external uniform magnetic and $\mathrm{AB}$ flux fields on a relativistic spinless particle (antiparticle) under equal mixture of scalar and vector Killingbeck potentials in Section 2. We discuss some special cases in Section 3. Finally, we give our concluding remarks in Section 4.

\section{The Klein-Gordon Atom}

The Klein-Gordon atom for the spinless particle with mass $m_{e}$ and charge $-e$ moving in external electromagnetic field and $\mathrm{AB}$ flux field given by potentials $V(r), S(r)$ and $\vec{A}$ reads $[40,41]$

$$
\begin{aligned}
& {\left[c^{2}\left(\vec{p}+\frac{e-\vec{A}}{c}\right)^{2}-(E-V(r))^{2}+\left(m_{e} c^{2}+S(r)\right)^{2}\right]} \\
& \times \psi(r, \phi)=0 .
\end{aligned}
$$

The scalar and vector potentials are chosen in the following form [27-30]:

$$
V_{K}(r)=\lambda r^{2}+\sigma r-\frac{\kappa}{r}, \quad \vec{A}=\frac{1}{2} \vec{B} \times \vec{r}+\frac{\Phi_{\mathrm{AB}}}{2 \pi r} \widehat{\phi},
$$

where $V_{K}(r)$ is the Killingbeck potential, that is, harmonic oscillator potential plus Cornell potential [35-38], which is extensively used in particle physics $[42,43]$. Moreover, the vector potential in the symmetric gauge is defined by $\vec{A}=$ $\vec{A}_{1}+\vec{A}_{2}$ such that $\vec{\nabla} \times \vec{A}_{1}=\vec{B}$ and $\vec{\nabla} \times \vec{A}_{2}=0$, where the applied magnetic field $\vec{B}=(0,0, B)$ is perpendicular to the plane of transversal motion of the particle and $\vec{A}_{2}$ describes the additional $\mathrm{AB}$ flux field $\Phi_{\mathrm{AB}}$ created by a solenoid in cylindrical coordinates [32]. The wave function in (1) is defined by

$$
\psi(r, \phi)=\frac{1}{\sqrt{2 \pi}} e^{i m \phi} \frac{R(r)}{\sqrt{r}}, \quad m=0, \pm 1, \pm 2, \ldots,
$$

where $m$ is the eigenvalue of angular momentum. The relationship between the attractive scalar and repulsive vector potentials is given by $S(r)=\beta V(r)$, where $-1 \leq \beta \leq$ 1 is arbitrary constant and hence the KG equation could be reduced to a Schrödinger-type second-order differential equation as follows:

$$
\begin{aligned}
& {\left[c^{2}\left(\vec{p}+\frac{e \vec{A}}{c}\right)^{2}+2\left(E V(r)+m_{e} c^{2} S(r)\right)\right.} \\
& \left.+S^{2}(r)-V^{2}(r)+m_{e}^{2} c^{4}-E^{2}\right] \psi(r, \phi)=0 .
\end{aligned}
$$

Now, we will treat the bound-state solutions of the two cases in (4) as follows. 
2.1. The Positive Energy Solution. The positive energy states require that $S(r)=V(r)$ (i.e., $\beta=1$ case) which, in the non-relativistic limit, corresponds to the solution of the wave equation:

$$
\begin{aligned}
& \left\{\frac{1}{2 \mu}\left[-i \hbar \vec{\nabla}+\frac{e}{c}\left(\frac{B r}{2}+\frac{\Phi_{\mathrm{AB}}}{2 \pi r}\right) \hat{\phi}\right]^{2}+2 V_{K}(r)-E\right\} \\
& \times \psi(r, \phi)=0,
\end{aligned}
$$

where $\psi(r, \phi)$ stands for non-relativistic wave function. Thus, the choice $S(r)=V(r)$ produces a non-relativistic limit with a potential function $2 V(r)$ and not $V(r)$. Accordingly, it would be natural to scale the potential term in (4) and (5) so that in the non-relativistic limit the interaction potential becomes $V(r)$ not $2 V(r)$. Thus, we need to recast (4) for the $S(r)=V(r)$ as $[32,39,40,44]$

$$
\begin{gathered}
{\left[c^{2}\left(-i \hbar \vec{\nabla}+\frac{e \vec{A}}{c}\right)^{2}+\left(E+m_{e} c^{2}\right) V(r)\right] \psi(r, \phi)} \\
=\left(E^{2}-m_{e}^{2} c^{4}\right) \psi(r, \phi),
\end{gathered}
$$

where

$$
\nabla^{2}=\frac{1}{\rho} \frac{\partial}{\partial \rho}+\frac{\partial^{2}}{\partial \rho^{2}}+\frac{1}{\rho^{2}} \frac{\partial^{2}}{\partial \phi^{2}}+\frac{\partial^{2}}{\partial z^{2}},
$$

and in order to simplify (6), we introduce new parameters $\alpha_{1}=E+m_{e} c^{2}$ and $\alpha_{2}=E-m_{e} c^{2}$ so that it can be reduced to the following form:

$$
\begin{aligned}
& \left\{c^{2}\left[-i \hbar \vec{\nabla}+\frac{e}{c}\left(\frac{B r}{2}+\frac{\Phi_{\mathrm{AB}}}{2 \pi r}\right) \hat{\phi}\right]^{2}-\alpha_{1}\left(\alpha_{2}-V_{K}(r)\right)\right\} \\
& \times \psi(r, \phi)=0 .
\end{aligned}
$$

By inserting (2) and (3) into (8), we obtain

$$
\begin{gathered}
\frac{d^{2} R(r)}{d r^{2}}+\frac{\alpha_{1}}{\hbar^{2} c^{2}}\left[\alpha_{2}-U_{\mathrm{eff}}\left(r, \omega_{L}, \xi\right)\right] R(r)=0, \\
U_{\mathrm{eff}}\left(r, \omega_{L}, \xi\right)=V_{K}(r)+\frac{m_{e}^{2} c^{2} \omega_{L}^{2}}{\alpha_{1}} r^{2} \\
+\frac{\hbar^{2} c^{2}}{\alpha_{1}} \frac{\left(m^{\prime 2}-1 / 4\right)}{r^{2}}+\frac{2 \hbar \omega_{L} m_{e} c^{2}}{\alpha_{1}} m^{\prime}, \\
\omega_{L}=\frac{\Omega}{2}, \quad \Omega=\frac{|e| B}{m_{e} c}, \quad m^{\prime}=m+\xi \\
m^{\prime}=1,2, \ldots, \quad \xi=\frac{\Phi_{\mathrm{AB}}}{\Phi_{0}},
\end{gathered}
$$

where the effective potentials depending on the magnitudes of two fields strength with $\omega_{L}$ and $m^{\prime}$ are the Larmor frequency and a new eigenvalue of angular momentum (magnetic quantum number), respectively. It is worthy to mention that the frequency $\Omega$ is called the cyclotron frequency [45]. This is the frequency of rotation corresponding to the classical motion of a charged particle in a uniform magnetic field and $\Omega / 2$ is the Larmor frequency in units of $\mathrm{Hz}$ [45]. Moreover, we take $\xi$ as integer with the flux quantum $\Phi_{0}=h c / e$. Here, $V_{K}(r)$ is a pure Killingbeck potential (2), the second term is the harmonic oscillator-type potential, and the other terms are the rotational potential creating the rotational energy levels. Equations (9a), (9b), and (9c) can be alternatively expressed as

$$
\begin{gathered}
\frac{d^{2} R(r)}{d r^{2}}+\left(\frac{C_{1}}{r^{2}}+\frac{C_{2}}{r}-C_{3}-C_{4} r-C_{5} r^{2}\right) R(r)=0, \\
C_{1}=-\left(m^{\prime 2}-\frac{1}{4}\right), \quad C_{2}=\frac{\alpha_{1} \kappa}{\hbar^{2} c^{2}} \\
C_{3}=\frac{2 m_{e} \omega_{L} m^{\prime}}{\hbar}-\frac{\alpha_{1} \alpha_{2}}{\hbar^{2} c^{2}} \\
C_{4}=\frac{\alpha_{1} \sigma}{\hbar^{2} c^{2}}, \quad C_{5}=\frac{\alpha_{1} \lambda}{\hbar^{2} c^{2}}+\left(\frac{m_{e} \omega_{L}}{\hbar}\right)^{2}
\end{gathered}
$$

with the asymptotic behaviors $R(0)=0$ and $R(\infty) \rightarrow 0$. It is interesting to look at the results obtained from (10a) for a special case $C_{2}=C_{4}=0$; that is, we have

$$
\frac{d^{2} R(r)}{d r^{2}}+\left(\frac{C_{1}}{r^{2}}-C_{5} r^{2}\right) R(r)=C_{3} R(r),
$$

which corresponds to the differential equation of a harmonic oscillator with a centrifugal term. It is a form similar to the one given by (7) in [46] with the equivalence $C_{1} \rightarrow-l^{\prime}\left(l^{\prime}+1\right)$, $C_{5} \rightarrow \alpha^{2}$ and $C_{3} \rightarrow-\lambda_{n}(\alpha, l$, and $\lambda$ are the parameters used in [46]). So by putting the parameter values

$$
\begin{gathered}
l^{\prime}=m^{\prime}-\frac{1}{2}, \quad \alpha=\sqrt{\frac{\left(E+m_{e} c^{2}\right) \lambda}{\hbar^{2} c^{2}}+\left(\frac{m_{e} \omega_{L}}{\hbar}\right)^{2}}, \\
\lambda_{n}=\frac{E^{2}-m_{e}^{2} c^{4}}{\hbar^{2} c^{2}}-\frac{2 m_{e} \omega_{L} m^{\prime}}{\hbar}
\end{gathered}
$$

into (14) of [45], we obtain the energy equation as

$$
\begin{aligned}
E^{2}-m_{e}^{2} c^{4}= & 2 m_{e} c^{2} \hbar \omega_{L} m^{\prime} \\
& +2 \hbar c^{2}\left(2 n+m^{\prime}+1\right) \\
& \times \sqrt{\frac{\left(E+m_{e} c^{2}\right) \lambda}{c^{2}}+\left(m_{e} \omega_{L}\right)^{2}} .
\end{aligned}
$$

Now, we find a solution to (10a) by making the following choice of the wave function $[37,38,47,48]$ :

$$
R_{n m}(r)=\exp \left(\frac{1}{2} p r^{2}+q r\right) \sum_{n=0}^{\infty} a_{n} r^{n+\delta},
$$

where $p$ and $q$ are parameters whose values are to be determined in terms of the potential parameters $\lambda, \sigma$, and $\kappa$. Substituting (14) into (10a), we obtain the series [36]

$$
\begin{gathered}
\sum_{n=0}^{\infty} a_{n} S_{n} r^{n+\delta-2}+\sum_{n=1}^{\infty} a_{n-1} T_{n-1} r^{n+\delta-2}+\sum_{n=2}^{\infty} a_{n-2} W_{n-2} r^{n+\delta-2}=0, \\
p^{2}=C_{5}, \quad 2 p q=C_{4},
\end{gathered}
$$


with

$$
\begin{gathered}
S_{n}=(n+\delta)(n+\delta-1)+C_{1}, \\
T_{n-1}=2 q(n+\delta-1)+C_{2}, \\
W_{n-2}=q^{2}+2 p\left(n+\delta-\frac{3}{2}\right)-C_{3},
\end{gathered}
$$

where the two parameters $p$ and $q$ are determined in order for the radial wave functions $R_{n m}(r)$ to be finite everywhere and vanish at $r=0$ and as $r \rightarrow \infty$. To obtain the recurrence relation which can connect various expansion coefficients $a_{n}$, we make identical powers of $r$ in (15), that is, equate the coefficients of $r^{n+\delta-2}$ to zero. Thus, the relations become

$$
\begin{gathered}
a_{0}\left[\delta(\delta-1)+C_{1}\right]=0 \Longrightarrow \delta^{2}+C_{1}=\delta, \\
a_{0} \neq 0 \Longrightarrow \delta= \pm m^{\prime}+\frac{1}{2}, \\
a_{1}=-\frac{\left(2 q \delta+C_{2}\right)}{2 \delta} a_{0}, \\
a_{2}=-\frac{\left[p(2 \delta+1)+q^{2}-C_{3}\right] a_{0}+\left[2 q(\delta+1)+C_{2}\right] a_{1}}{2(2 \delta+1)}, \\
a_{3}=-\left(\frac{\left(2 p q-C_{4}\right) a_{0}+\left[p(2 \delta+3)+q^{2}-C_{3}\right] a_{1}}{6(\delta+1)}\right. \\
\left.+\frac{\left[2 q(\delta+2)+C_{2}\right] a_{2}}{6(\delta+1)}\right), \\
a_{n}=-\left(\frac{\left(p^{2}-C_{5}\right) a_{n-4}+\left(2 p q-C_{4}\right) a_{n-3}}{n(2 \delta+n-1)}\right. \\
+\frac{\left[p(2 \delta+2 n-3)+q^{2}-C_{3}\right] a_{n-2}}{n(2 \delta+n-1)} \\
\left.+\frac{\left[2 q(\delta+n-1)+C_{2}\right] a_{n-1}}{n(2 \delta+n-1)}\right),
\end{gathered}
$$

where $n=0,1,2, \ldots$, with $a_{0} \neq 0$. Here, the positive sign of parameter $\delta=m^{\prime}+1 / 2$ has been selected. The power series for large values of $\delta$ or $\mathrm{m}^{\prime}$ is convergent. For convenience, we take the ratio of two successive terms, that is, $a_{n+1} / a_{n}$, which becomes

$$
\begin{aligned}
\frac{a_{1}}{a_{0}}= & -\left(q+\frac{C_{2}}{2 \delta}\right) \longrightarrow-q \quad \text { when } \delta \longrightarrow \infty \\
\frac{a_{2}}{a_{0}}= & \frac{q^{2}\left(\delta+C_{2} / 2 q\right)\left[\delta+C_{2} / 2 q+1\right]}{\delta(2 \delta+1)} \\
& -\frac{\left[p(2 \delta+1)+q^{2}-C_{3}\right]}{2(2 \delta+1)} \longrightarrow \frac{q^{2}-p}{2}
\end{aligned}
$$

when $\delta \longrightarrow \infty$.
It is apparent from the above relations that the power series converges to zero when $\delta \rightarrow \infty$. Hence, the series must be truncated (bounded) for $n=n_{\max }$. At this value of $n$, we obtain the following equations:

$$
\begin{aligned}
p= & \pm \sqrt{\frac{\left(E+m_{e} c^{2}\right) \lambda}{\hbar^{2} c^{2}}+\left(\frac{m_{e} \omega_{L}}{\hbar}\right)^{2}} \\
\Longrightarrow & p=-\sqrt{\frac{\left(E+m_{e} c^{2}\right) \lambda}{\hbar^{2} c^{2}}+\left(\frac{m_{e} \omega_{L}}{\hbar}\right)^{2}}, \quad p \neq 0, \\
2 p q= & C_{4} \\
\Longrightarrow & q=-\frac{\left(E+m_{e} c^{2}\right) \sigma}{2 \hbar^{2} c^{2} \sqrt{\left(\left(E+m_{e} c^{2}\right) \lambda / \hbar^{2} c^{2}\right)+\left(m_{e} \omega_{L} / \hbar\right)^{2}}}, \\
\frac{(E+}{\left.m_{e} c^{2}\right)\left(E-m_{e} c^{2}\right)} & \frac{\left(E+m_{e} c^{2}\right) \kappa}{\hbar^{2} c^{2}}=-2 q\left(n+m^{\prime}-\frac{1}{2}\right), \\
= & \frac{2 m_{e} \omega_{L} m^{\prime}}{\hbar}+2 \sqrt{\frac{\left(E+m_{e} c^{2}\right) \lambda}{\hbar^{2} c^{2}}+\left(\frac{m_{e} \omega_{L}}{\hbar}\right)^{2}} \\
& \times\left(m^{\prime}+n-1\right)-q^{2},
\end{aligned}
$$

where the negative sign of the coefficient $p$ has been chosen in (19a). It is worth noting that the potential parameter $\lambda$ must be positive when $\omega_{L}=0$. Hence, (19c) gives a restriction on the potential parameters $\lambda, \sigma$, and $\kappa$ as follows:

$$
\sigma=\frac{\kappa}{\left(n+m^{\prime}-1 / 2\right)} \sqrt{\frac{\left(E+m_{e} c^{2}\right) \lambda}{\hbar^{2} c^{2}}+\left(\frac{m_{e} \omega_{L}}{\hbar}\right)^{2}},
$$

and it follows that (19d) gives the energy formula as

$$
\begin{aligned}
E^{2}-m_{e}^{2} c^{4}= & 2 m_{e} c^{2} \hbar \omega_{L} m^{\prime}+2 \hbar^{2} c^{2}\left(n+m^{\prime}-1\right) \\
& \times \sqrt{\frac{\left(E+m_{e} c^{2}\right) \lambda}{\hbar^{2} c^{2}}+\left(\frac{m_{e} \omega_{L}}{\hbar}\right)^{2}} \\
& -\frac{\left(E+m_{e} c^{2}\right)^{2} \kappa^{2}}{4 \hbar^{2} c^{2}\left(n+m^{\prime}-1 / 2\right)^{2}},
\end{aligned}
$$

where $n=0,1,2, \ldots$. We may find a solution to the above transcendental equation as $E=E_{\mathrm{KG}}^{(+)}=E_{n m^{\prime}}\left(\omega_{L}, \xi\right)$. Overmore, the wave function (14) with the help of (17), (19a), and (19b) becomes 


$$
\begin{gathered}
\psi_{n, m}^{(+)}(r, \phi)=C_{n, m} \frac{1}{\sqrt{2 \pi}} e^{i m \phi} r^{m+\xi} e^{-(1 / 2) \sqrt{\left(E+m_{e} c^{2}\right) \lambda / \hbar^{2} c^{2}+\left(m_{e} \omega_{L} / \hbar\right)^{2}}\left[r^{2}+\left(\left(E+m_{e} c^{2}\right) \sigma /\left(\left(E+m_{e} c^{2}\right) d_{2}+\hbar^{2} c^{2}\left(m_{e} \omega_{L} / \hbar\right)^{2}\right)\right) r\right]} \sum_{n=0}^{n_{\max }} a_{n} r^{n}, \\
a_{1}=-a_{0}\left[q+\frac{\left(E+m_{e} c^{2}\right) \kappa}{2 \hbar^{2} c^{2}\left(m^{\prime}+1 / 2\right)}\right], \\
a_{2}=-\frac{1}{2} a_{0}\left[p-q^{2}-\frac{m_{e} \omega_{L} m^{\prime}}{\hbar\left(m^{\prime}+1\right)}-\frac{\left(E+m_{e} c^{2}\right) \kappa}{\hbar^{2} c^{2}\left(m^{\prime}+1 / 2\right)}\left(q+\frac{\left(E+m_{e} c^{2}\right) \kappa}{4 \hbar^{2} c^{2}\left(m^{\prime}+1\right)}\right)\right],
\end{gathered}
$$

where $C_{n, m}$ is the normalization constant.

In the non-relativistic limit, when $E+m_{e} c^{2} \rightarrow 2 \mu$, $E+m_{e} c^{2} \rightarrow E_{n m^{\prime}}$ and $c=1,(21)$ and (22a) give the energy formula

$$
\begin{aligned}
E_{n m^{\prime}}\left(\omega_{L}, \xi\right)= & \hbar \omega_{L} m^{\prime}+\hbar\left(n+m^{\prime}-1\right) \\
& \times \sqrt{\frac{2 \lambda}{\mu}+\omega_{L}^{2}}-\frac{\mu \kappa^{2}}{2 \hbar^{2}\left(n+m^{\prime}-1 / 2\right)^{2}},
\end{aligned}
$$

and wave function

$$
\begin{aligned}
\psi_{n, m}^{(+)}(r, \phi)= & C_{n, m} \frac{1}{\sqrt{2 \pi}} e^{i m \phi} r^{m+\xi} \\
& \times e^{-(\mu / 2 \hbar) \sqrt{2 \lambda / \mu+\omega_{L}^{2}}\left[r^{2}+\left(2 \sigma / \mu\left(2 d_{2} / \mu+\omega_{L}^{2}\right)\right) r\right]} \sum_{n=0}^{n_{\max }} a_{n} r^{n},
\end{aligned}
$$

respectively, for the Schrödinger-Killingbeck system with the following restriction on the potential parameters $\lambda, \sigma$, and $\kappa$ given by

$$
\sigma=\frac{\mu \kappa}{\hbar\left(n+m^{\prime}-1 / 2\right)} \sqrt{\frac{2 \lambda}{\mu}+\omega_{L}^{2}} .
$$

Notice that the present model has been solved in $2 \mathrm{D}$ space with an external uniform magnetic field since it is perpendicular to the plane, where the vector and scalare Cornell potentials have the dimensions of $r=\rho$ and $\phi$; that is, $V=S=V(\rho, \phi)[32]$. However, without the magnetic field, the model can be solved in any desired dimensional space by considering the change $m \rightarrow l+(D-2) / 2[49,50]$, where $D$ refers to spatial dimension and also the existence of interdimensional degeneracy.

As shown in Figure 1(a) and (9b), the effective potential function changes in shape when the magnetic field strength increases, say $\omega_{L}=8$ and in absence of $\mathrm{AB}$ flux field. The energy levels are raised when the strength of the magnetic field increases and in absence of $\mathrm{AB}$ flux field $\xi=0$. We see that the effective potential changes gradually from the pure pseudo-harmonic oscillator potential, when $\omega_{L}=0$, to a pure harmonic oscillator type behavior in short potential range when $\omega_{L}=8$. In Figure 1(b), the effective potential (9b) which is pseudo-harmonic oscillator when $\omega_{L}=0$ becomes sensitive to the increasing $\mathrm{AB}$ flux field $\xi=8$ in the short range region; that is, $0<r<4$ a.u.

2.2. The Bound States for Negative Energy. In this case of $S(r)=-V(r)$, the first inspection of (4) shows that the following changes $V(r) \rightarrow-V(r)$, (i.e., $\lambda \rightarrow-\lambda, \sigma \rightarrow-\sigma$, $\kappa \rightarrow-\kappa), \psi_{n, m}^{(+)}(r, \phi) \rightarrow \psi_{n, m}^{(-)}(r, \phi)$ and $E \rightarrow-E$ give the negative energy solution for antiparticles as

$$
\begin{aligned}
E^{2}- & m_{e}^{2} c^{4}-2 m_{e} c^{2} \hbar \omega_{L} m^{\prime} \\
= & 2 \hbar^{2} c^{2}\left(n+m^{\prime}-1\right) \sqrt{\frac{\left(E-m_{e} c^{2}\right) \lambda}{\hbar^{2} c^{2}}+\left(\frac{m_{e} \omega_{L}}{\hbar}\right)^{2}} \\
& -\frac{\left(E-m_{e} c^{2}\right)^{2} \kappa^{2}}{4 \hbar^{2} c^{2}\left(n+m^{\prime}-1 / 2\right)^{2}},
\end{aligned}
$$

with restriction on potential parameters

$$
\sigma=\frac{\kappa}{\left(n+m^{\prime}-1 / 2\right)} \sqrt{\frac{\left(E-m_{e} c^{2}\right) \lambda}{\hbar^{2} c^{2}}+\left(\frac{m_{e} \omega_{L}}{\hbar}\right)^{2}} .
$$

We may find solution to the above transcendental equation as $E=E_{K G}^{(-)}=E_{n m^{\prime}}\left(\omega_{L}, \xi\right)$. Overmore, the wave function for antiparticle reads 


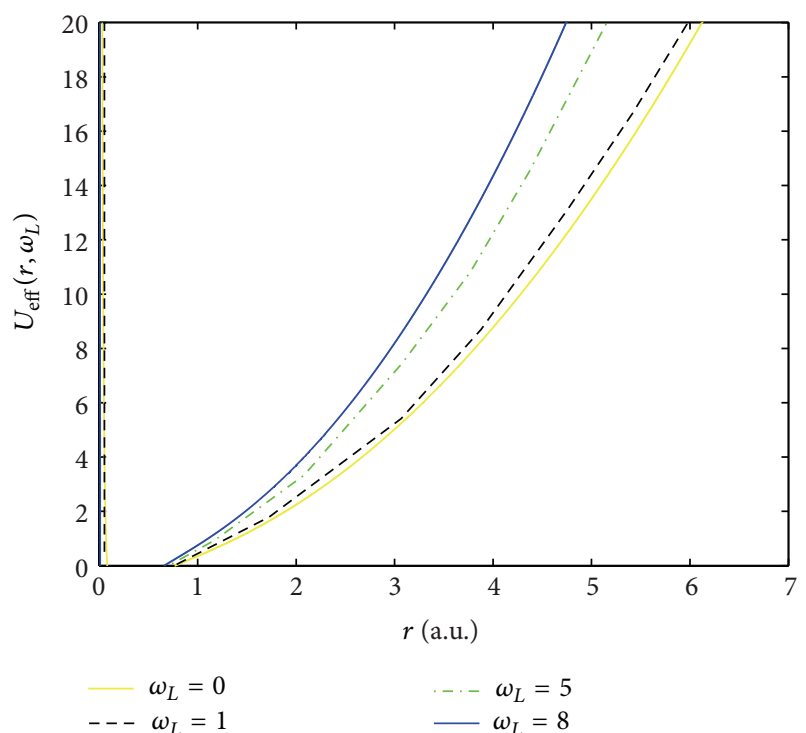

(a)

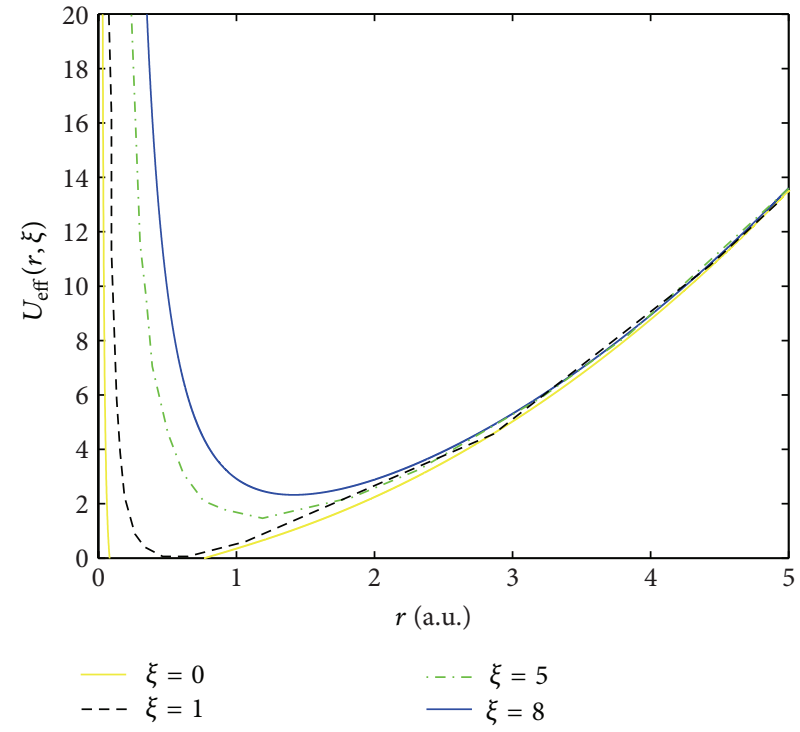

(b)

Figure 1: The KG effective potential function for (a) $\omega_{L}=0,1,5,8$ and with $\xi=0$. (b) $\xi=0,1,5,8$ and with $\omega_{L}=0$ (colour online). Here, $m_{e}=\hbar=c=1$.

$$
\begin{gathered}
\psi_{n, m}^{(-)}(r, \phi)=D_{n, m} \frac{1}{\sqrt{2 \pi}} e^{i m \phi} r^{m+\xi} e^{-(1 / 2) \sqrt{\left(E-m_{e} c^{2}\right) \lambda / \hbar^{2} c^{2}+\left(m_{e} \omega_{L} / \hbar\right)^{2}}\left[r^{2}+\left(\left(E-m_{e} c^{2}\right) \sigma /\left(\left(E-m_{e} c^{2}\right) d_{2}+\hbar^{2} c^{2}\left(m_{e} \omega_{L} / \hbar\right)^{2}\right)\right) r\right]} \sum_{n=0}^{n_{\max }} a_{n} r^{n} \\
a_{1}=-a_{0}\left[q+\frac{\left(E-m_{e} c^{2}\right) \kappa}{2 \hbar^{2} c^{2}\left(m^{\prime}+1 / 2\right)}\right] \\
a_{2}=-\frac{1}{2} a_{0}\left[p-q^{2}-\frac{m_{e} \omega_{L} m^{\prime}}{\hbar\left(m^{\prime}+1\right)}-\frac{\left(E-m_{e} c^{2}\right) \kappa}{\hbar^{2} c^{2}\left(m^{\prime}+1 / 2\right)}\left(q+\frac{\left(E-m_{e} c^{2}\right) \kappa}{4 \hbar^{2} c^{2}\left(m^{\prime}+1\right)}\right)\right]
\end{gathered}
$$

where $D_{n, m}$ is the normalization constant.

\section{Discussions}

In this section, we discuss some special cases of interest from our general solution.

(i) If we set $\sigma=\kappa=0$, the effective Killingbeck potential turns to effective harmonic oscillator potential in relativistic case as

$$
\begin{aligned}
U_{\mathrm{eff}}\left(r, \omega_{L}, \xi\right)= & \lambda r^{2}+\frac{m_{e}^{2} c^{2} \omega_{L}^{2}}{\left(E+m_{e} c^{2}\right)} r^{2} \\
& +\frac{\hbar^{2} c^{2}}{\left(E+m_{e} c^{2}\right)} \frac{\left(m^{\prime 2}-1 / 4\right)}{r^{2}} \\
& +\frac{2 \hbar \omega_{L} m_{e} c^{2} m^{\prime}}{\left(E+m_{e} c^{2}\right)}, \quad \lambda=\frac{1}{2} \mu \omega^{2} .
\end{aligned}
$$

The bound state solutions (with the change $n=2\left(n_{r}+1\right)$ ) are $[32,36]$

$$
\begin{aligned}
E^{2}-m_{e}^{2} c^{4}= & 2 m_{e} c^{2} \hbar \omega_{L} m^{\prime}+4 \hbar c^{2}\left(n_{r}+\frac{m^{\prime}+1}{2}\right) \\
& \times \sqrt{\frac{\left(E+m_{e} c^{2}\right) \lambda}{c^{2}}+\left(m_{e} \omega_{L}\right)^{2}}
\end{aligned}
$$

$$
\begin{aligned}
\psi_{n_{r}, m}^{(+)}(r, \phi)= & A_{n_{r}, m} \frac{1}{\sqrt{2 \pi}} e^{i m \phi} r^{m+\xi} \\
& \times e^{-(1 / 2) \sqrt{\left(E+m_{e} c^{2}\right) \lambda / \hbar^{2} c^{2}+\left(m_{e} \omega_{L} / \hbar\right)^{2}} r^{2}} \sum_{n=0}^{n_{\max }} a_{n} r^{2\left(n_{r}+1\right)},
\end{aligned}
$$

$$
a_{1}=0
$$

$$
a_{2}=\frac{1}{2} a_{0}\left[\sqrt{\frac{\left(E+m_{e} c^{2}\right) \lambda}{\hbar^{2} c^{2}}+\left(\frac{m_{e} \omega_{L}}{\hbar}\right)^{2}}+\frac{m_{e} \omega_{L} m^{\prime}}{\hbar\left(m^{\prime}+1\right)}\right] \text {, }
$$


where $A_{n_{r}, m}$ is the normalization constant. On the other hand, the nonrelativistic effective harmonic oscillator potential

$$
U_{\mathrm{eff}}\left(r, \omega_{L}, \xi\right)=\lambda r^{2}+\frac{1}{2} \omega_{L}^{2} r^{2}+\frac{\left(m^{\prime 2}-1 / 4\right) \hbar^{2}}{2 m_{e} r^{2}}+\hbar \omega_{L} m^{\prime}
$$

has bound states as [32]

$$
\begin{aligned}
E_{n m^{\prime}}\left(\omega_{L}, \xi\right)= & \hbar \omega_{L} m^{\prime} \\
& +2 \hbar \Omega^{\prime}\left(n_{r}+\frac{\left|m^{\prime}\right|+1}{2}\right), \Omega^{\prime}=\sqrt{\omega_{L}^{2}+\omega^{2}}, \\
\psi_{n_{r}, m}^{(+)}(r, \phi)= & C_{n, m} \frac{1}{\sqrt{2 \pi}} e^{i m \phi} r^{m+\xi} e^{-(\mu / 2 \hbar) \Omega^{\prime} r^{2}} \\
& \times \sum_{n=0}^{n_{\max }} a_{n} r^{2\left(n_{r}+1\right)}, \quad n_{r}=0,1,2, \ldots, \\
= & \frac{1}{\sqrt{2 \pi}} e^{i m \phi} \sqrt{\frac{2 b^{m^{\prime}+1} n !}{\left(n+m^{\prime}\right) !} r^{m^{\prime}} e^{-b r^{2} / 2} L_{n}^{\left(m^{\prime}\right)}\left(b r^{2}\right),} \\
a_{1}= & 0, \\
a_{2}=\frac{\mu}{2 \hbar} \Omega^{\prime} a_{0}, \quad a_{0} \neq 0 . & \quad(36 \mathrm{~b})
\end{aligned}
$$

(ii) Setting $\lambda=\sigma=0$ in (21), the killingbeck potential turns to the Coulomb potential case (i.e., $V(r)=-A / r$ and $\left.n=n_{r}+1\right)$. Thus, we obtain the relativistic energy formula

$$
\begin{aligned}
E^{2}-m_{e}^{2} c^{4}= & 2 m_{e} c^{2}\left(n_{r}+2 m^{\prime}\right) \hbar \omega_{L} \\
& -\frac{\left(E+m_{e} c^{2}\right)^{2} \kappa^{2}}{4 \hbar^{2} c^{2}\left(n_{r}+m^{\prime}+1 / 2\right)^{2}}, \quad \kappa=Z e^{2},
\end{aligned}
$$

and the wave function

$$
\begin{gathered}
\psi_{n_{r}, m}^{(+)}(r, \phi)=C_{n_{r}, m} \frac{1}{\sqrt{2 \pi}} e^{i m \phi} r^{m+\xi} e^{-\left(m_{e} \omega_{L}\right) / 2 \hbar r^{2}} \\
\times \sum_{n=0}^{n_{\max }} a_{n} r^{n_{r}+1}, \\
a_{1}=-\frac{\left(E+m_{e} c^{2}\right) \kappa}{2 \hbar^{2} c^{2}\left(m^{\prime}+1 / 2\right)} a_{0}, \\
a_{2}=-\frac{1}{2}\left[\frac{m_{e} \omega_{L}}{\hbar}-\frac{m_{e} \omega_{L} m^{\prime}}{\hbar\left(m^{\prime}+1\right)}\right. \\
\left.-\frac{\left(E+m_{e} c^{2}\right)^{2} \kappa^{2}}{4 \hbar^{4} c^{4}\left(m^{\prime}+1\right)\left(m^{\prime}+1 / 2\right)}\right] a_{0} .
\end{gathered}
$$

Overmore, in the nonrelativistic limit, we have

$$
E=\hbar \omega_{L} m^{\prime}+\hbar\left(n_{r}+m^{\prime}\right) \omega_{L}-\frac{m_{e} Z^{2} e^{4}}{2 \hbar^{2}\left(n_{r}+m^{\prime}+1 / 2\right)^{2}},
$$

and the wave function becomes

$$
\begin{gathered}
\psi_{n_{r}, m}^{(+)}(r, \phi)=C_{n_{r}, m} \frac{1}{\sqrt{2 \pi}} e^{i m \phi} r^{m+\xi} e^{-\left(\mu \omega_{L} / 2 \hbar\right) r^{2}} \sum_{n=0}^{n_{\max }} a_{n} r^{n_{r}+1}, \\
a_{1}=-\frac{m_{e} A}{\hbar^{2}\left(m^{\prime}+1 / 2\right)} a_{0}, \\
a_{2}=-\frac{1}{2}\left[\frac{\mu \omega_{L}}{\hbar}-\frac{\mu \omega_{L} m^{\prime}}{\hbar\left(m^{\prime}+1\right)}-\frac{\mu^{2} Z^{2} e^{4}}{\hbar^{4}\left(m^{\prime}+1\right)\left(m^{\prime}+1 / 2\right)}\right] a_{0} .
\end{gathered}
$$

Notice that in the absence of external fields $\omega_{L}=\xi=0$, the problem can be solved in any dimension. Thus, applying the transformation $|m| \rightarrow l+1 / 2$, our previous results are reduced to the well-known three-dimensional bound state solutions for the harmonic oscillator and the hydrogen-like atoms in the Coulomb fields [40]:

$$
\begin{gathered}
E^{ \pm}= \pm m_{e} c^{2}\left[1+\frac{2 \hbar c}{m_{e} c^{2}}\left(2 n_{r}+l+\frac{3}{2}\right) \sqrt{\left.\frac{\left(E+m_{e} c^{2}\right) \lambda}{m_{e}^{2} c^{4}}\right]^{1 / 2},}\right. \\
E^{ \pm}= \pm m_{e} c^{2}\left[1-\frac{Z^{2} e^{4}}{4 \hbar^{2} c^{2}\left(n_{r}+l+1\right)^{2}}\left(1+\frac{E}{m_{e} c^{2}}\right)^{2}\right]^{1 / 2},
\end{gathered}
$$

respectively. According to (41), the energy spectrum can be found for the scalar particle and antiparticle.

In Figure 2(a), we plot the effective potential for the case of low vibrational $(n=0,1,2,3)$ and rotational $(m=1)$ levels for various Larmor frequencies $\omega_{L}=0,1,5,8$ and $\xi=0$ case. As shown in Figure 2(a) and (31), the effective potential function changes in shape as well as the bound state energy eigenvalues increase when $\omega_{L}=8$. It is shown that the energy levels are raised when the strength of the magnetic field increases and in the absence of $\mathrm{AB}$ flux field. It is also obvious that the effective potential changes gradually from the pure pseudo-harmonic oscillator potential, which is a nonmagnetic $\left(\omega_{L}=0\right)$ and $\mathrm{AB}$ flux $(\xi=0)$ fields case, to a pure harmonic oscillator type behavior in short potential range when the strength of the applied magnetic field is increased to $\omega_{L}=8$. If we consider a strong magnetic field case $\omega_{L}=8$ which has the shape of pure harmonic oscillator potential function, the energy difference between adjacent energy levels is nearly equal which is a known characteristic of the pure harmonic oscillator potential. In Figure 2(b), we plot the effective potential (34) in the absence of the magnetic field and in the presence of $\mathrm{AB}$ flux field in the short range region.

In Tables 1 and 2, we show the effect of magnetic field and $\mathrm{AB}$ flux field, respectively, on the low vibrational $n$ 


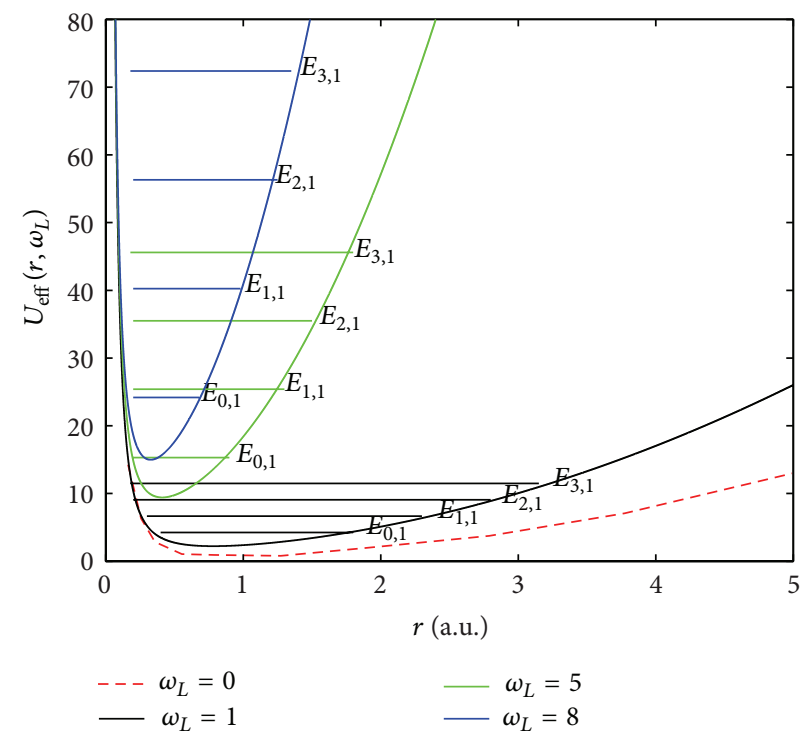

(a)

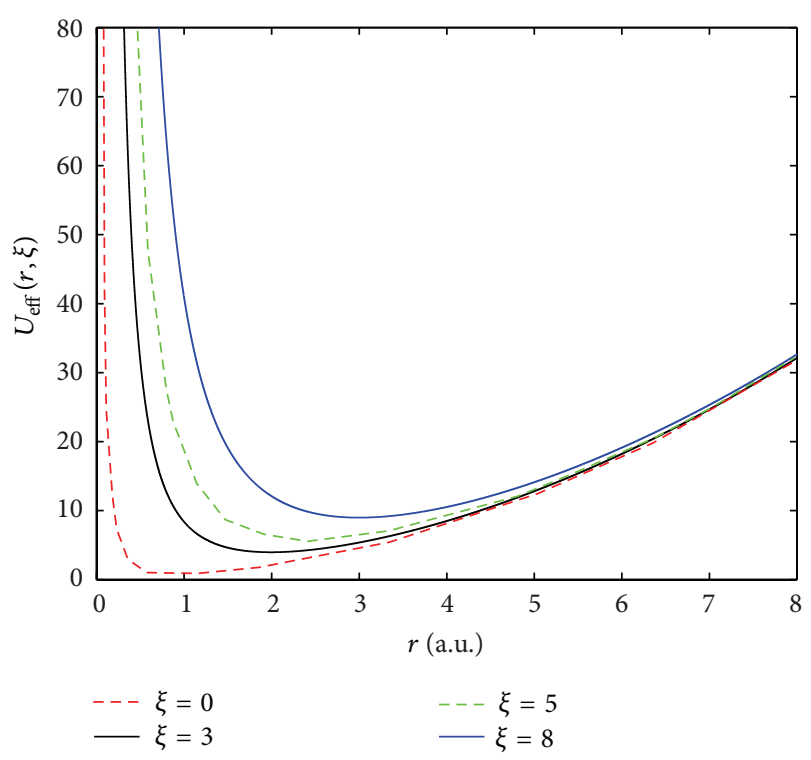

(b)

FIGURE 2: The Schrödinger effective potential function and corresponding bound state energy levels $\left(E_{n m}\right)$ in low vibrational $(n=0,1,2,3)$ and rotational $(m=1)$ levels for (a) $\omega_{L}=0,1,5,8$ with $\xi=0$. (b) $\xi=0,1,5,8$ and with $\omega_{L}=0$ (color online). Here, $\mu=\hbar=1$.

and rotational $m$ relativistic energy states of the harmonic oscillator potential. As shown in Table 1, when the magnetic field is not applied and without $\mathrm{AB}$ flux field $\left(\omega_{L}=0\right.$, $\xi=0$ ), the spacing between the energy levels of the effective potential is narrow and decreases with increasing $n$. But when the magnetic field strength increases, the energy levels of the effective potential increase and the spacings between states also increase. In Table 2, when the AB flux field is applied and without magnetic field, the energy states become degenerate for various values of $n$ and $m$ and for various $A B$ flux field strength values. In Tables 3 and 4, we show the effect of magnetic field and $A B$ flux field, respectively, on the low vibrational $n$ and rotational $m$ nonrelativistic energy states of the harmonic oscillator potential. As shown in Table 3, when the magnetic field is not applied and without $\mathrm{AB}$ flux field $\left(\omega_{L}=0, \xi=0\right)$, the energy states are equally spaced (the pure harmonic oscillator case). But when the magnetic field strength is raised, the energy levels of the effective potential increase and the spacings between states also increase. In Table 4, when the AB flux field is applied and without magnetic field, the energy states become degenerate and equally spaced for various values of $n$ and $m$ and for various $\mathrm{AB}$ flux field strength values.

\section{Concluding Remarks}

To sum up, in this paper, we have studied the solution of two-dimensional KG and Schrödinger equations with the Killingbeck potential for low vibrational and rotational energy levels without and with a constant magnetic field having arbitrary Larmor frequeny and $\mathrm{AB}$ flux field. We have applied the wave function ansatz method for $\omega_{L} \neq 0$ (with magnetic field) and $\xi \neq 0$ (with $\mathrm{AB}$ flux field) to obtain analytical expressions, in closed form, for bound state energies and wave functions of the spinless relativistic particle subject to a Killingbeck interaction expressed in terms of external uniform magnetic and $A B$ flux fields in any vibrational $n$ and rotational $m$ states. The above results show that the problems of relativistic quantum mechanics can be also solved exactly as in the non-relativistic ones. We considered the solution of both positive (particle) and negative (antiparticle) KG energy states. It is noticed that the solution with equal mixture of scalar-vector potentials can be easily reduced into the well-known Schrödinger solution for a particle with an interaction potential field and a free field, respectively. We have also studied the bound-state solutions for some special cases including the non-relativistic limits (Schrödinger equation for harmonic oscillator and Coulomb potentials under external magnetic and $A B$ flux fields) and also the KG equation for harmonic oscillator and Coulomb interactions. The results show that the splitting is not constant and is mainly dependent on the strength of the external magnetic field and $A B$ flux field. In order to show the effect of constant magnetic and $\mathrm{AB}$ flux fields on the vibrational and rotational energy levels of the harmonic oscillator, we plot the effective potential and corresponding energy levels with increasing Larmor frequency and flux field for special potential parameters. We have seen that the effective potential function and corresponding energy levels are raised in energy when magnetic and $\mathrm{AB}$ flux field strengths increase. The effective potential function behavior gradually changes from the pure pseudo-harmonic oscillator to a pure harmonic oscillator shape in short potential range as the magnetic and $\mathrm{AB}$ flux fields strengths increase. 
TABLE 1: The KG energy eigenvalues ( $E_{n m}$ in atomic units) of the harmonic oscillator potential for various $\omega_{L}$ values and $\xi=0$. Here, $\hbar=c=$ $m_{e}=k=1$.

\begin{tabular}{ccccccccccc}
\hline$m$ & $n$ & & & \multicolumn{9}{c}{$E_{n m}$} \\
& & $\omega_{L}=0$ & $\omega_{L}=1$ & $\omega_{L}=2$ & $\omega_{L}=3$ & $\omega_{L}=4$ & $\omega_{L}=5$ & $\omega_{L}=6$ & $\omega_{L}=7$ & $\omega_{L}=8$ \\
\hline & 0 & 1.83929 & 2.04353 & 2.40325 & 2.75615 & 3.08137 & 3.38066 & 3.65815 & 3.91752 & 4.16166 \\
0 & 1 & 3.09625 & 3.4289 & 4.03986 & 4.65113 & 5.21808 & 5.74094 & 6.22602 & 6.67941 & 7.10609 \\
& 2 & 4.12383 & 4.51488 & 5.26359 & 6.03138 & 6.75146 & 7.4193 & 8.04087 & 8.62301 & 9.17158 \\
& 3 & 5.03104 & 5.45806 & 6.30283 & 7.18744 & 8.02542 & 8.80667 & 9.53605 & 10.2205 & 10.8663 \\
\hline & 0 & 2.50976 & 3.16597 & 3.89307 & 4.55265 & 5.14478 & 5.68284 & 6.17802 & 6.63858 & 7.0706 \\
1 & 1 & 3.62919 & 4.27262 & 5.12444 & 5.93706 & 6.68087 & 7.36315 & 7.99437 & 8.58337 & 9.13707 \\
& 2 & 4.58916 & 5.22885 & 6.16824 & 7.09549 & 7.95634 & 8.7516 & 9.49036 & 10.1815 & 10.8323 \\
& 3 & 5.45354 & 6.09216 & 7.09891 & 8.11893 & 9.0768 & 9.96689 & 10.7966 & 11.5745 & 12.3081 \\
\hline
\end{tabular}

TABLE 2: The KG energy eigenvalues ( $E_{n m}$ in atomic units) of the harmonic oscillator potential for various $\xi$ values and $\omega_{L}=0$.

\begin{tabular}{ccccccccccc}
\hline$m$ & $n$ & & & & \multicolumn{3}{c}{$E_{n m}$} \\
& & $\xi=0$ & $\xi=1$ & $\xi=2$ & $\xi=3$ & $\xi=4$ & $\xi=5$ & $\xi=6$ & $\xi=7$ & $\xi=8$ \\
\hline \multirow{4}{*}{0} & 0 & 1.83929 & 2.50976 & 3.09625 & 3.62919 & 4.12383 & 4.58916 & 5.03104 & 5.45354 & 5.85966 \\
& 1 & 3.09625 & 3.62919 & 4.12383 & 4.58916 & 5.03104 & 5.45354 & 5.85966 & 6.25166 & 6.6313 \\
& 2 & 4.12383 & 4.58916 & 5.03104 & 5.45354 & 5.85966 & 6.25166 & 6.63137 .0 & 7.0 & 7.35892 \\
& 3 & 5.03104 & 5.45354 & 5.85966 & 6.25166 & 6.6313 & 7.0 & 7.35892 & 7.70901 & 8.05108 \\
\hline & 0 & 2.50976 & 3.09625 & 3.62919 & 4.12383 & 4.58916 & 5.03104 & 5.45354 & 5.85966 & 6.25166 \\
1 & 1 & 3.62919 & 4.12383 & 4.58916 & 5.03104 & 5.45354 & 5.85966 & 6.25166 & 6.6313 & 7.0 \\
& 2 & 4.58916 & 5.03104 & 5.45354 & 5.85966 & 6.25166 & 6.6313 & 7.0 & 7.35892 & 7.70901 \\
& 3 & 5.45354 & 5.85966 & 6.25166 & 6.6313 & 7.0 & 7.35892 & 7.70901 & 8.05108 & 8.38582 \\
\hline
\end{tabular}

TABLE 3: The nonrelativistic energy eigenvalues ( $E_{n m}$ in atomic units) of the harmonic oscillator potential for various $\omega_{L}$ values and $\xi=0$.

\begin{tabular}{ccccccccccc}
\hline$m$ & $n$ & & & \multicolumn{9}{c}{$E_{n m}$} \\
& & $\omega_{L}=0$ & $\omega_{L}=1$ & $\omega_{L}=2$ & $\omega_{L}=3$ & $\omega_{L}=4$ & $\omega_{L}=5$ & $\omega_{L}=6$ & $\omega_{L}=7$ & $\omega_{L}=8$ \\
\hline & 0 & 1.0 & 1.41421 & 2.23607 & 3.16228 & 4.12311 & 5.09902 & 6.08276 & 7.07107 & 8.06226 \\
0 & 1 & 3.0 & 4.24264 & 6.7082 & 9.48683 & 12.3693 & 15.2971 & 18.2483 & 21.2132 & 24.1868 \\
& 2 & 5.0 & 7.07107 & 11.1803 & 15.8114 & 20.6155 & 25.4951 & 30.4138 & 35.3553 & 40.3113 \\
& 3 & 7.0 & 9.89949 & 15.6525 & 22.1359 & 28.8617 & 35.6931 & 42.5793 & 49.4972 & 56.4358 \\
\hline & 0 & 2.0 & 3.82843 & 6.47214 & 9.32456 & 12.2462 & 15.198 & 18.1655 & 21.1421 & 24.1245 \\
1 & 1 & 4.0 & 6.65685 & 10.9443 & 15.6491 & 20.4924 & 25.3961 & 30.3311 & 35.2843 & 40.249 \\
& 2 & 6.0 & 9.48528 & 15.4164 & 21.9737 & 28.7386 & 35.5941 & 42.4966 & 49.4264 & 56.3735 \\
& 3 & 8.0 & 12.3137 & 19.8885 & 28.2982 & 36.9848 & 45.7922 & 54.6621 & 63.5685 & 72.4981 \\
\hline
\end{tabular}

TABLE 4: The nonrelativistic energy eigenvalues ( $E_{n m}$ in atomic units) of the harmonic oscillator potential for various $\xi$ values and $\omega_{L}=0$.

\begin{tabular}{|c|c|c|c|c|c|c|c|c|c|c|}
\hline \multirow{2}{*}{$m$} & \multirow{2}{*}{$n$} & \multicolumn{9}{|c|}{$E_{n m}$} \\
\hline & & $\xi=0$ & $\xi=1$ & $\xi=2$ & $\xi=3$ & $\xi=4$ & $\xi=5$ & $\xi=6$ & $\xi=7$ & $\xi=8$ \\
\hline \multirow{4}{*}{0} & 0 & 1.0 & 2.0 & 3.0 & 4.0 & 5.0 & 6.0 & 7.0 & 8.0 & 9.0 \\
\hline & 1 & 3.0 & 4.0 & 5.0 & 6.0 & 7.0 & 8.0 & 9.0 & 10.0 & 11.0 \\
\hline & 2 & 5.0 & 6.0 & 7.0 & 8.0 & 9.0 & 10.0 & 11.0 & 12.0 & 13.0 \\
\hline & 3 & 7.0 & 8.0 & 9.0 & 10.0 & 11.0 & 12.0 & 13.0 & 14.0 & 15.0 \\
\hline \multirow{4}{*}{1} & 0 & 2.0 & 3.0 & 4.0 & 5.0 & 6.0 & 7.0 & 8.0 & 9.0 & 10.0 \\
\hline & 1 & 4.0 & 5.0 & 6.0 & 7.0 & 8.0 & 9.0 & 10.0 & 11.0 & 12.0 \\
\hline & 2 & 6.0 & 7.0 & 8.0 & 9.0 & 10.0 & 11.0 & 12.0 & 13.0 & 14.0 \\
\hline & 3 & 8.0 & 9.0 & 10.0 & 11.0 & 12.0 & 13.0 & 14.0 & 15.0 & 16.0 \\
\hline
\end{tabular}




\section{Acknowledgments}

One of the authors (Sameer M. Ikhdair) would like to thank the president of An-Najah National University (ANU), Professor Rami Alhamdallah, and the acting president Professor Maher Natsheh for their continuous support during his present work at ANU. The authors acknowledge the partial support of the Scientific and Technological Research Council of Turkey.

\section{References}

[1] W. Greiner and B. Muller, Quantum Mechanics: An Introduction, Springer, Berlin, Germany, 1994.

[2] S. Özçelik and M. Şimşek, "Exact solutions of the radial Schrödinger equation for inverse-power potentials," Physics Letters A, vol. 152, no. 3-4, pp. 145-150, 1991.

[3] S.-H. Dong, "Schrödinger equation with the potential $V(r)=$ $\mathrm{Ar}^{-4}+\mathrm{Br}^{-3}+\mathrm{Cr}^{-2}+\mathrm{Dr}^{-1}$, Physica Scripta, vol. 64, no. 4, pp. 273-276, 2001.

[4] S.-H. Dong and Z.-Q. Ma, "Exact solutions to the Schrödinger equation for the potential $V(r)=a r^{2}+b r^{-4}+c r^{-6}$ in two dimensions," Journal of Physics A, vol. 31, no. 49, pp. 9855-9859, 1998.

[5] M. S. Child, S.-H. Dong, and X.-G. Wang, "Quantum states of a sextic potential: hidden symmetry and quantum monodromy," Journal of Physics A, vol. 33, no. 32, pp. 5653-5661, 2000.

[6] S.-H. Dong, "Exact solutions of the two-dimensional Schrödinger equation with certain central potentials," International Journal of Theoretical Physics, vol. 39, no. 4, pp. 1119-1128, 2000.

[7] S.-H. Dong, "A new approach to the relativistic schrödinger equation with central potential: Ansatz method," International Journal of Theoretical Physics, vol. 40, no. 2, pp. 559-567, 2001.

[8] S.-H. Dong, "On the solutions of the Schrödinger equation with some anharmonic potentials: wave function ansatz," Physica Scripta, vol. 65, no. 4, pp. 289-295, 2002.

[9] S. Flugge, Practical Quantum Mechanics 1, Springer, Berlin, Germany, 1994.

[10] X.-Q. Zhao, C.-S. Jia, and Q.-B. Yang, "Bound states of relativistic particles in the generalized symmetrical double-well potential," Physics Letters A, vol. 337, no. 3, pp. 189-196, 2005.

[11] S. M. Ikhdair, "Exact solution of Dirac equation with charged harmonic oscillator in electric field: bound states," Journal of Modern Physics, vol. 3, no. 2, pp. 170-179, 2012.

[12] W. C. Qiang, "Bound states of the Klein-Gordon equation for ring-shaped Kratzer-type potential," Chinese Physics, vol. 13, no. 5, pp. 575-578, 2004.

[13] S. Ikhdair and R. Sever, "Exact polynomial eigensolutions of the Schrödinger equation for the pseudoharmonic potential," Journal of Molecular Structure, vol. 806, no. 1-3, pp. 155-158, 2007.

[14] F.-L. Lu, C.-Y. Chen, and D.-S. Sun, "Bound states of KleinGordon equation for double ring-shaped oscillator scalar and vector potentials," Chinese Physics, vol. 14, no. 3, pp. 463-467, 2005.

[15] S. M. Ikhdair and R. Sever, "Exact solutions of the Ddimensional Schrödinger equation for a ring-shaped pseudoharmonic potential," Central European Journal of Physics, vol. 6, no. 3, pp. 685-696, 2008.

[16] S. M. Ikhdair and R. Sever, "Exact bound states of the Ddimensional Klein-Gordon equation with equal scalar and vector ring-shaped pseudoharmonic potential," International Journal of Modern Physics C, vol. 19, no. 9, pp. 1425-1442, 2008.

[17] S. M. Ikhdair and R. Sever, "Exactly solvable effective mass d-dimensional schrödinger equation for pseudoharmonic and modified kratzer problems," International Journal of Modern Physics C, vol. 20, no. 3, pp. 361-372, 2009.

[18] R.-C. Wang and C.-Y. Wong, "Finite-size effect in the Schwinger particle-production mechanism," Physical Review D, vol. 38, no. 1, pp. 348-359, 1988.

[19] J. N. Ginocchio, "A relativistic symmetry in nuclei," Physics Report, vol. 315, no. 1-4, pp. 231-240, 1999.

[20] A. Bohr, I. Hamarnoto, and B. R. Motelson, "Pseudospln in rotating nuclear potentials," Physica Scripta, vol. 26, no. 4, pp. 267-272, 1982.

[21] J. Dudek, W. Nazarewicz, Z. Szymanski, and G. A. Leander, "Abundance and systematics of nuclear superdeformed states; relation to the pseudospin and pseudo-SU(3) symmetries," Physical Review Letters, vol. 59, no. 13, pp. 1405-1408, 1987.

[22] A. Arima, M. Harvey, and K. Shimizu, "Pseudo LS coupling and pseudo SU3 coupling schemes," Physics Letters B, vol. 30, no. 8, pp. 517-522, 1969.

[23] K. T. Hecht and A. Adler, "Generalized seniority for favored $J$ \# 0 pairs in mixed configurations," Nuclear Physics A, vol. 137, no. 1, pp. 129-143, 1969.

[24] P. R. Page, T. Goldman, and J. N. Ginocchio, "Relativistic symmetry suppresses quark spin-orbit splitting," Physical Review Letters, vol. 86, no. 2, pp. 204-207, 2001.

[25] S. Weinberg, The Quantum Theory of Fields, vol. 1, Cambridge University Press, Cambridge, UK, 1995.

[26] D. Boyanovsky, C. Destri, and H. J. de Vega, "Approach to thermalization in the classical $\varphi^{4}$ theory in $1+1$ dimensions: energy cascades and universal scaling," Physical Review D, vol. 69, no. 4, Article ID 045003, 2004.

[27] R. Khordad, "Effects of magnetic field and geometrical size on the interband light absorption in a quantum pseudodot system," Solid State Sciences, vol. 12, no. 7, pp. 1253-1256, 2010.

[28] R. Khordad, "Simultaneous effects of temperature and pressure on the donor binding energy in a V-groove quantum wire," Superlattices and Microstructures, vol. 47, no. 3, pp. 422-431, 2010.

[29] A. Çetin, "A quantum pseudodot system with a twodimensional pseudoharmonic potential," Physics Letters A, vol. 372, no. 21, pp. 3852-3856, 2008.

[30] S. M. Ikhdair, M. Hamzavi, and R. Sever, "Spectra of cylindrical quantum dots: the effect of electrical and magnetic fields together with AB flux field," Physica B, vol. 407, pp. 4523-4529, 2012.

[31] S. M. Ikhdair and M. Hamzavi, "A quantum pseudodot system with two-dimensional pseudoharmonic oscillator in external magnetic and Aharonov-Bohm fields," Physica B, vol. 407, pp. 4198-4207, 2012.

[32] S. M. Ikhdair and M. Hamzavi, "Effects of extermal fields on a two-dimensional Klein-Gordon particle under pseudoharmonic oscillator interaction," Chinese Physics B, vol. 21, no. 11, pp. 110302-110306, 2012.

[33] M. Aygun, O. Bayrak, I. Boztosun, and Y. Sahin, “The energy eigenvalues of the Kratzer potential in the presence of a magnetic field," European Physical Journal D, vol. 66, no. 2, Article ID 20319, 2012.

[34] S. M. Ikhdair and M. Hamzavi, "Spectral properties of quantum dots influenced by a confining potential model," Physica B, vol. 407, pp. 4797-4803, 2012. 
[35] M. Hamzavi and A. A. Rajabi, "Solution of Dirac equation with Killingbeck potential by using wave function ansatz method under spin symmetry limit," Communications in Theoretical Physics, vol. 55, no. 1, pp. 35-37, 2011.

[36] M. Hamzavi, S. M. Ikhdair, and K. E. Thylwe, "Pseudospin symmetry in the relativistic Killingbeck potential: Quasi-exact solution," Zeitschrift für Naturforschung A, vol. 67, pp. 567-571, 2012.

[37] R. Kumar and F. Chand, "Series solution to the N-dimensional radial Schrödinger equation for the quark-antiquark interaction," Physica Scripta, vol. 85, no. 5, pp. 055008-055004, 2012.

[38] R. Kumar and F. Chand, "Reply to comments on: series solution to the $\mathrm{N}$-dimensionalradial Schrödinger equation for the quark-antiquark interaction," Physica Scripta, vol. 86, no. 2, p. 027001, 2012.

[39] S. M. Ikhdair, "A scalar charged particle in presence of magnetic and Aharonov-Bohm field plus scalar-vector Killingbeck potentials," Few-Body Systems, vol. 54, no. 11, pp. 1987-1995, 2013.

[40] W. Greiner, Relativistic Quantum Mechanics: Wave Equations, Springer, Berlin, Germany, 2000.

[41] A. D. Alhaidari, H. Bahlouli, and A. Al-Hasan, "Dirac and Klein-Gordon equations with equal scalar and vector potentials," Physics Letters A, vol. 349, no. 1-4, pp. 87-97, 2006.

[42] G. Plante and A. F. Antippa, "Analytic solution of the Schrödinger equation for the Coulomb-plus-linear potential," Journal of Mathematical Physics, vol. 46, no. 6, pp. 1-20, 2005.

[43] J. Killingbeck, "Perturbation theory without wavefunctions," Physics Letters A, vol. 65, no. 2, pp. 87-88, 1978.

[44] Y. Xu, S. He, and C.-S. Jia, "Approximate analytical solutions of the Klein-Gordon equation with the Pöschl-Teller potential including the centrifugal term," Physica Scripta, vol. 81, no. 4, Article ID 045001, 2010.

[45] R. L. Liboff, Introductory Quantum Mechanics, Pearson Education-Addison Wesley, San Francisco, Calif, USA, 2003.

[46] G. Chen, Z.-D. Chen, and Z.-M. Lou, "Bound states of the KleinGordon and Dirac equation for scalar and vector pseudoharmonic oscillator potentials," Chinese Physics, vol. 13, no. 3, pp. 279-282, 2004.

[47] S. H. Dong, Z. Ma, and G. Espozito, "Exact solutions of the Schrödinger equation with inversepowerpotential," Foundations of Physics Letters, vol. 12, no. 5, pp. 465-474, 1999.

[48] A. Arda and R. Sever, "Effective-mass Klein-Gordon equation for non-PT/non-Hermitian generalized Morse potential," Physica Scripta, vol. 82, no. 6, Article ID 065007, 2010.

[49] S. M. Ikhdair and J. Abu-Hasna, "Quantization rule solution to the Hulthén potential in arbitrary dimension with a new approximate scheme for the centrifugal term," Physica Scripta, vol. 83, no. 2, Article ID 025002, 2011.

[50] S. M. Ikhdair and R. Sever, "Exact quantization rule to the Kratzer-type potentials: An application to the diatomic molecules," Journal of Mathematical Chemistry, vol. 45, no. 4, pp. 1137-1152, 2009. 

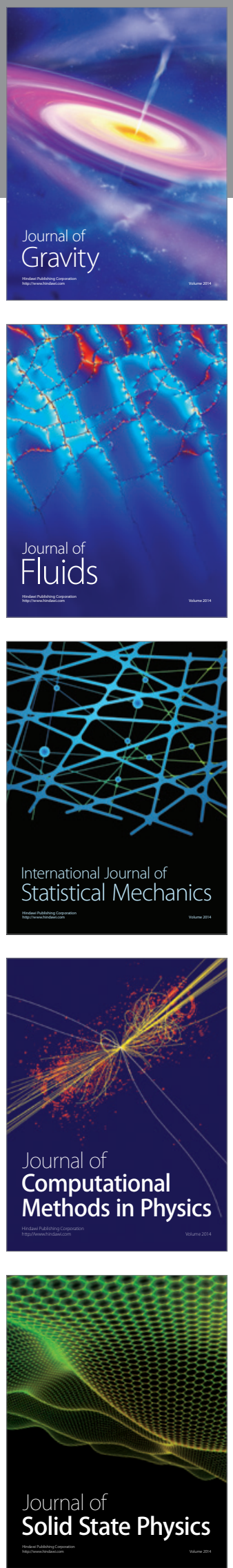

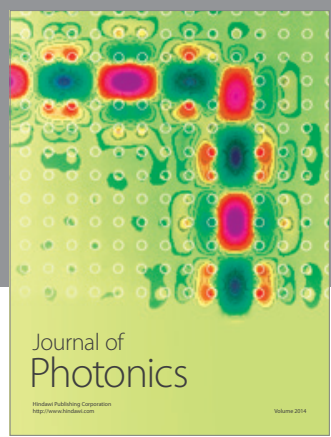

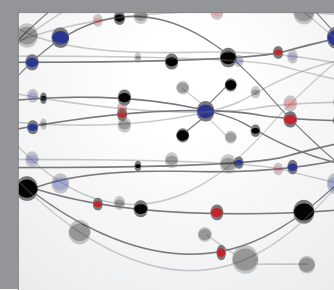

The Scientific World Journal

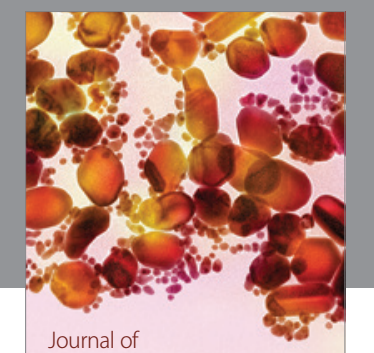

Soft Matter
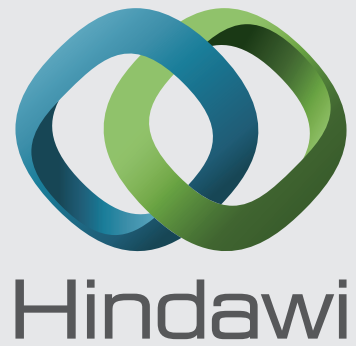

Submit your manuscripts at

http://www.hindawi.com
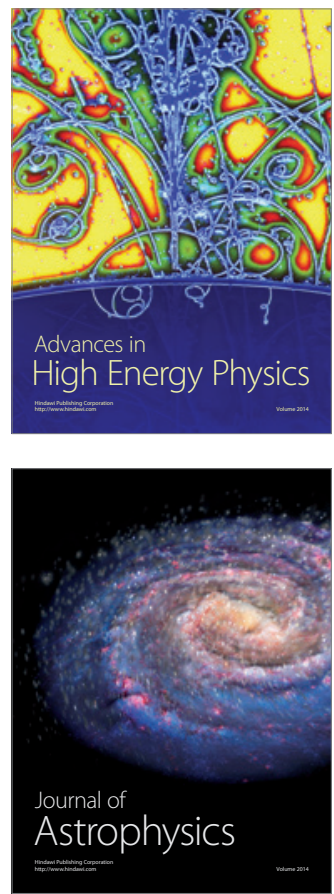
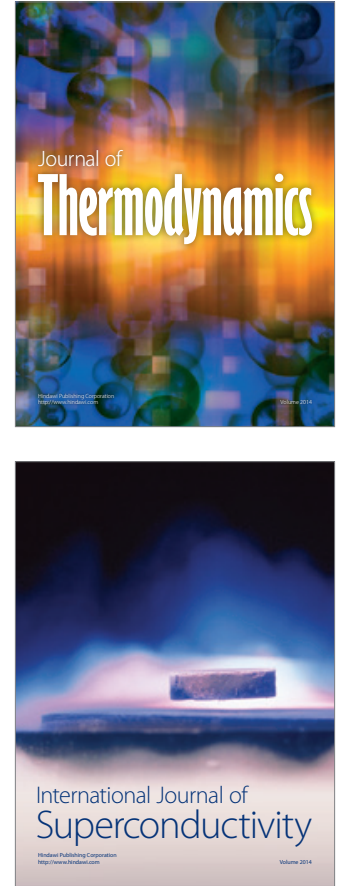
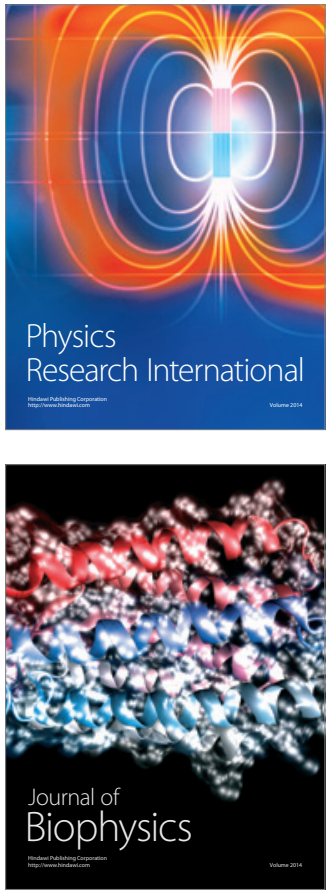
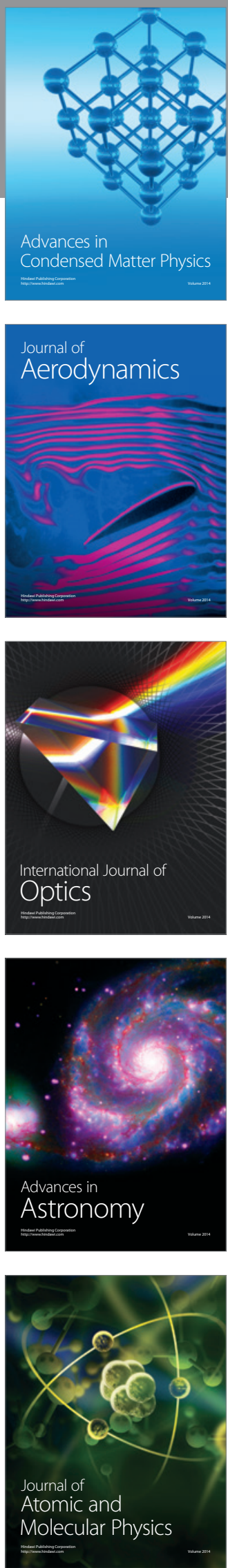